\title{
BAYESIAN ASYMPTOTICS WITH MISSPECIFIED MODELS
}

\author{
Pierpaolo De Blasi ${ }^{1,2}$ and Stephen G. Walker ${ }^{3}$ \\ ${ }^{1}$ University of Torino, ${ }^{2}$ Collegio Carlo Alberto and ${ }^{3}$ University of Kent
}

\begin{abstract}
In this paper, we study the asymptotic properties of a sequence of posterior distributions based on an independent and identically distributed sample and when the Bayesian model is misspecified. We find a sufficient condition on the prior for the posterior to accumulate around the densities in the model closest in the Kullback-Leibler sense to the true density function. Examples are presented.
\end{abstract}

Key words and phrases: Asymptotics, consistency, misspecified model.

\section{Introduction}

This paper is concerned with asymptotics for Bayesian nonparametric models. In particular, we consider generalizations of the recent literature on consistency; see for example, Barron, Schervish, and Wasserman ([199.9), Ghosal, Ghosh, and Ramamoorthi (1199.9), and Walker (2004). The standard assumption for consistency is that the true density function, which we denote by $f_{0}$, is in the Kullback-Leibler support of the prior, denoted by $\Pi$. Further sufficient conditions on the prior are then established in order to ensure that the sequence of posterior distributions accumulate in suitable neighborhoods of $f_{0}$. The three papers just cited deviate in the precise form of the further sufficient conditions.

We make the support of the prior assumption more general now by assuming that the closest density in the support of the prior is a possibly non-zero Kullback-Leibler divergence away from $f_{0}$; specifically, if $f_{1}$ is the closest density, in the Kullback-Leibler sense, in the support $\mathcal{F}$ of the prior (to be made more precise later), then $\delta_{1}$ is defined to be the Kullback-Leibler divergence between $f_{0}$ and $f_{1}$. We then look for further sufficient conditions under which the posterior distributions accumulate in suitable neighborhoods of $f_{1}$. In particular, we work around the ideas presented in Walker (2004) and the sufficient conditions for accumulation at $f_{1}$ can be seen as a generalization of the condition appearing in Walker (20104).

The convenience of working in this setting is quite evident. When considering asymptotics, there are two possible scenarios: $\delta_{1}=0$ or $\delta_{1}>0$. The former involves a well specified model and the latter a misspecified model. Typically, the latter is more likely, though in reality it will be unknown. However, one can 
assume $\delta_{1}=0$ and derive conditions on the prior for posterior accumulation at $f_{0}$, and then assume $\delta_{1}>0$ and derive another set of conditions on the prior for posterior accumulation at $f_{1}$. It is to be sure that the latter conditions will be stronger than the former. In this case, and the value of $\delta_{1}$ unknown, it makes perfect sense to construct the prior under the condition that $\delta_{1} \geq 0$, and hence under the misspecified case.

Early work for the misspecified problem has been done by Berk (1966) and more recently by Bunke and Milhaud (11998), Shalizil (200.9), and Kleijn and van der Vaart (20106). In particular, the strategy followed by Kleijn and van der vaart (2006) consists of defining a neighborhood around $f_{1}$ according to a suitable semimetric on the space of densities that satisfies an entropy condition related to the Hellinger integral $h_{\alpha}$ (to be defined later); see their equation (2.2). Kleijn and van der Vaart concentrate on the notion of a single $f_{1}$ for which accumulation of the posterior takes place and extend this to a finite number of such $f_{1}$.

On the other hand, we focus our efforts directly on a set $\mathcal{F}_{1}$ rather than on a single $f_{1}$, acknowledging the fact that in general one does not know how big the set of densities associated with the minimum Kullback-Leibler distance $\delta_{1}$ is. Hence, we find it appropriate to define

$$
\mathcal{F}_{1}=\left\{f \in \overline{\mathcal{F}}: D\left(f_{0}, f\right) \leq \delta_{1}\right\}
$$

where $\overline{\mathcal{F}}$ is the Hellinger closure of $\mathcal{F}$ and $D\left(f_{0}, f\right)$ is the Kullback-Leibler divergence of $f$ relative to $f_{0}$. When $\mathcal{F}_{1}$ is non-empty, our working assumption throughout the paper, we show accumulation at $\mathcal{F}_{1}$ with respect to the Hellinger distance. When $\mathcal{F}_{1}$ is empty, we show that the posterior accumulates in a different set that we define and explain at the end of Section 3.

In reality, and in general, it is not known whether $\mathcal{F}_{1}$ is empty or not, since $f_{0}$ is not known. A notable exception is when $\mathcal{F}$ is convex, in which case $\mathcal{F}_{1}$ reduces to a single density $f_{1}$. However, our key prior condition, given in Section 3 , covers both $\mathcal{F}_{1}$ empty or non-empty. Kleijn and van der Vaart (2006) only establish what the posterior does when $\mathcal{F}_{1}$ is a finite set. For a chosen $\Pi$ it may be possible to find $C_{\Pi}$ such that if $f_{0} \in C_{\Pi}$ then $\mathcal{F}_{1}$ is empty, whereas if $f_{0} \in C_{\Pi}^{c}$ then $\mathcal{F}_{1}$ is non-empty. But in spite of this being a difficult task, it would not even be known if $f_{0}$ was in $C_{\Pi}$ or not, and hence the objective in this area would focus on finding $\Pi$ for which $C_{\Pi}$ can be shown to be empty. To date this is only known to be true when $\mathcal{F}$ is convex. Therefore knowing what happens when $f_{0} \in C_{\Pi}$ is important, and one of the contributions of the paper is to fill this gap in the literature.

The rest of the paper is organized as follows. In Section 2 we start with some notation, definitions, and preliminary results. The main results are presented in 
Section 3 and illustrations involving various priors are given in Section 4 . We conclude with a discussion in Section 5 .

\section{Notation and Preliminary Results}

We introduce the notation of the paper, together with essential preliminary results. Let $\mathbb{X}$ be a separable metric space endowed with its Borel $\sigma$-field $\mathscr{B}$, and denote by $\Omega$ the space of density functions on $(\mathbb{X}, \mathscr{B})$ relative to some reference measure (that is omitted henceforth for ease of notation). For $d$ a distance on $\Omega$ and $A \subset \Omega$, we denote by $N(\delta, A, d)$ the minimum number of balls of radius at most $\delta$, with respect to the metric $d$, needed to cover $A$. In particular, on $\Omega$ we consider the Hellinger distance $H(f, g)=\left\{\int(\sqrt{f}-\sqrt{g})^{2}\right\}^{1 / 2}$ (which makes $\Omega$ a separable space) and the Kullback-Leibler divergence $D(f, g)=\int \log (f / g) f$. Moreover, we define, for $\alpha \in(0,1)$, the Hellinger integral $h_{\alpha}(f, g)=\int f^{1-\alpha} g^{\alpha}$ and the $\alpha$-divergence $d_{\alpha}(f, g)=\alpha^{-1}\left[1-h_{\alpha}(f, g)\right]$, see Liese and Vajda (20106) and the references therein. We recall here that $h_{\alpha}(f, g) \leq 1$ for any $f, g \in \Omega$ and that $d_{\alpha}(f, g)$ is decreasing in $\alpha$ with $\lim _{\alpha \rightarrow 0} d_{\alpha}(f, g)=D(f, g)$ whenever $D(f, g)<\infty$. The case $\alpha=1 / 2$ yields the Hellinger distance, since $d_{1 / 2}(f, g)=H^{2}(f, g)$. A lemma, whose proof is deferred to the Appendix, provides a useful inequality that relates the Hellinger distance and the Hellinger integral $h_{\alpha}$. It is used in the proofs of Theorem 1 and Lemma 2.

Lemma 1. For any $f, g, f_{0} \in \Omega$ and $0 \leq \alpha \leq 1 / 2$,

$$
\left|h_{\alpha}\left(f_{0}, f\right)-h_{\alpha}\left(f_{0}, g\right)\right| \leq[H(f, g)]^{2 \alpha} \text {. }
$$

Note that for $\alpha=1 / 2$, we have $H(f, g) \geq\left|H^{2}\left(f_{0}, f\right)-H^{2}\left(f_{0}, g\right)\right| / 2$, which is weaker than the standard triangle inequality, as it can be proved by using (A.2) in the Appendix and the fact that $H(f, g) \leq \sqrt{2}$.

As we are going to deal with convergence of sets of densities, we consider the metric space $(\Omega, H)$, and define $H(A, f)=\inf _{g \in A} H(g, f)$ to be the Hellinger distance between $A$ and $f \in \Omega$, and $H(A, B)=\max \left\{\sup _{f \in B} H(A, f), \sup _{f \in A} H(B\right.$, $f)\}$ to be the Hausdorff distance (relative to the Hellinger) between $A$ and $B$. In particular, it can be shown that

$$
H(A, f) \leq H(B, f)+H(A, B) .
$$

Since the Hellinger is a bounded distance, convergence in the Hausdorff metric of a sequence $\left(A_{n}\right)$ to $A$ is equivalent to $H\left(A_{n}, f\right) \rightarrow H(A, f)$ for every $f \in \Omega$ (known as Kuratowski convergence). Moreover, in case of a decreasing sequence $\left(A_{n}\right)$, the limit is given by $\bigcap_{n} \overline{A_{n}}$, see Rockafellar and Wets (200.9, Chap. 4).

Now let $X_{1}, X_{2}, \ldots$ be independent and identically distributed random variables taking values in $(\mathbb{X}, \mathscr{B})$ with common density function $f_{0} \in \Omega$ and, given 
$\mathcal{F} \subseteq \Omega$, let $\Pi$ be a prior probability measure on $\mathcal{F}$. The Bayesian posterior measure is given by

$$
\Pi_{n}(A)=\frac{\int_{A} R_{n}(f) \Pi(\mathrm{d} f)}{\int_{\mathcal{F}} R_{n}(f) \Pi(\mathrm{d} f)},
$$

where $A$ is a measurable subset of $\mathcal{F}$ and $R_{n}(f)=\prod_{i=1}^{n} f\left(X_{i}\right) / f_{0}\left(X_{i}\right)$. We denote by $F_{0}^{\infty}$ the infinite product measure relative to $f_{0}$. Finally, upon definition of $\delta_{1}=\inf _{f \in \mathcal{F}} D\left(f_{0}, f\right)$, we refer to $\mathcal{F}_{1}$ in $(\mathbb{L} . \mathbb{d})$ as the set of pseudo-true densities $f_{1}$. An associate editor has suggested an alternative definition of the minimum Kullback-Leibler distance as $\delta_{1}=\inf \left\{t: \Pi\left(f: D\left(f_{0}, f\right) \leq t\right)>0\right\}$; we explicitly work with the former but note the latter is effectively equivalent for the purposes of our paper, see also (3.5) below. Existence of a pseudo-true density is a delicate issue. Given the lower semicontinuity of $D\left(f_{0}, \cdot\right)$ as a map from the metric space $(\Omega, H)$ to $\mathbb{R}$, see Lemma 8.2 in Kleijn and van der Vaart (2006), a (rather) stringent sufficient condition is the compactness of $\mathcal{F}$. To our knowledge, most of the theoretical results are based on the hypothesis of convexity of $\mathcal{F}, \sup _{f \in \mathcal{F}} \int \log (f) f_{0}$ being finite the essential additional requirement. See Liese and Vajda (1987, Chap. 8), Pfanzag】 (1990) and Patilea (2001).

\section{Main Results}

In the well-specified case with $\delta_{1}=0$, strong consistency corresponds to

$$
\Pi_{n}\left(f: H\left(f_{0}, f\right)>\epsilon\right) \rightarrow 0 \quad F_{0}^{\infty}-\text { a.s.. }
$$

for any $\epsilon>0$, entailing that the posterior concentrates all the mass in an arbitrarily small Hellinger neighborhood of the true $f_{0}$. In the misspecified case, it is reasonable to ask that the posterior concentrates mass around the set of pseudo-true densities $\mathcal{F}_{1}$ in (ㅁ. $)$,

$$
\Pi_{n}\left(\left\{f: H\left(\mathcal{F}_{1}, f\right)>\epsilon\right\}\right) \rightarrow 0 \quad F_{0}^{\infty}-\text { a.s.. }
$$

In order to establish this result, we follow a route different from the one in Kleijn and van der Vaart (2006) in that we keep on working, although in an instrumental way, on neighborhoods around the true $f_{0}$. Specifically, we consider the sets

$$
\begin{aligned}
A_{\alpha, \epsilon} & =\left\{f \in \mathcal{F}: \quad d_{\alpha}\left(f_{0}, f\right)>\delta_{1}+\frac{\epsilon}{\alpha}\right\}, \\
A_{\alpha} & =\left\{f \in \mathcal{F}: \quad d_{\alpha}\left(f_{0}, f\right)>\delta_{1}+\alpha\right\} .
\end{aligned}
$$

Note that the latter can be recovered from (B.2) with $\epsilon=\alpha^{2}$, although any $\epsilon=\epsilon(\alpha)$ decreasing in $\alpha$ with $\epsilon(\alpha) / \alpha \rightarrow 0$ as $\alpha \rightarrow 0$ would work. The idea is that $A_{\alpha}^{c}$ is monotonically decreasing in $\alpha$ to $\mathcal{F}_{1}$. 
Lemma 2. Let $A_{\alpha}$ and $\mathcal{F}_{1}$ be as in (区.3) and (메), respectively. Then, $\bigcap_{\alpha} \overline{A_{\alpha}^{c}} \subseteq$ $\mathcal{F}_{1}$.

The proof of Lemma 2 is provided in the Appendix. We aim now at establishing sufficient conditions for

$$
\Pi_{n}\left(A_{\alpha, \epsilon}\right) \rightarrow 0 \quad F_{0}^{\infty}-\text { a.s. }
$$

for any $\alpha$ and $\epsilon$ sufficiently small. To this aim, we first adapt the Kullback-Leibler property to the misspecified case as

$$
\Pi\left(f \in \mathcal{F}: \quad D\left(f_{0}, f\right) \leq \delta_{1}+\eta\right)>0
$$

for any $\eta>0$, see Theorem 2.1 in Kleijn and van der Vaart (2006). In fact, a simple corollary of Lemma 3 and 4 in Barron, Schervish, and Wasserman (II999) implies that, for all large $n$ and for any $c>0$,

$$
I_{n} \geq \mathrm{e}^{-n\left(\delta_{1}+c\right)}, \quad F_{0}^{\infty}-\text { a.s. }
$$

where $I_{n}=\int_{\mathcal{F}} R_{n}(f) \Pi(\mathrm{d} f)$ is the denominator of ([2.2). As for the numerator, the key condition can be stated, similar to Walker (2004), in terms of summability of powers of prior probabilities. To this end, for a given $\alpha \in(0,1)$, let $\left(B_{j, \varepsilon}\right)_{j \geq 1}$ be Hellinger balls of size $\varepsilon>0$ that cover $\mathcal{F}$ such that

$$
\sum_{j \geq 1} \Pi\left(B_{j, \varepsilon}\right)^{\alpha}<\infty
$$

We are now ready to state and prove our main result.

Theorem 1. Suppose $\Pi$ satisfies (B.5) and that (B.7) holds for some $\alpha \in(0,1 / 2)$, where the sets $B_{j, \varepsilon}$ are Hellinger balls of size $\varepsilon=2(\epsilon / 2)^{1 /(2 \alpha)}$ whose union covers $\mathcal{F}$. Then (B.4) holds.

Proof. Let $\left(A_{j}\right)_{j \geq 1}$ be a partition of $A_{\alpha, \epsilon}$ (to be specified later) and define $f_{n, j}$ to be the predictive density with posterior distribution restricted, and normalized, to the set $A_{j}$. Note that

$$
f_{n, j}(x)=\int_{A_{j}} f(x) \frac{\Pi_{n}(\mathrm{~d} f)}{\Pi_{n}\left(A_{j}\right)}=\frac{\int_{A_{j}} f(x) R_{n}(f) \Pi(\mathrm{d} f)}{\int_{A_{j}} R_{n}(f) \Pi(\mathrm{d} f)},
$$

so that, letting $L_{n, j}=\int_{A_{j}} R_{n}(f) \Pi(\mathrm{d} f)$,

$$
\frac{L_{n+1, j}}{L_{n, j}}=\frac{f_{n, j}\left(X_{n+1}\right)}{f_{0}\left(X_{n+1}\right)},
$$


see also Walker (20104). Then, we have

$$
\begin{aligned}
\Pi_{n}\left(A_{\alpha, \epsilon}\right) & =\sum_{j \geq 1} \Pi_{n}\left(A_{j}\right) \leq \sum_{j \geq 1} \Pi_{n}\left(A_{j}\right)^{\alpha}=\sum_{j \geq 1} \frac{L_{n, j}^{\alpha}}{I_{n}^{\alpha}}, \\
\mathrm{E}\left(L_{n+1, j}^{\alpha} \mid X_{1}, \ldots, X_{n}\right) & =h_{\alpha}\left(f_{0}, f_{n, j}\right) L_{n, j}^{\alpha} .
\end{aligned}
$$

Take $A_{j} \subseteq A_{j}^{*}=\left\{f: H\left(f_{j}, f\right)<\varepsilon / 2\right\}$, where $\varepsilon=2(\epsilon / 2)^{1 /(2 \alpha)}$ and $\left(f_{j}\right)_{j \geq 1}$ are densities in $A_{\alpha, \epsilon}$. By using Lemma 1, we have that

$$
h_{\alpha}\left(f_{0}, f_{n, j}\right)-h_{\alpha}\left(f_{0}, f_{j}\right) \leq\left[H\left(f_{j}, f_{n, j}\right)\right]^{2 \alpha}<\frac{\epsilon}{2}
$$

which, together with $h_{\alpha}\left(f_{0}, f_{j}\right)<1-\alpha\left(\delta_{1}+\epsilon / \alpha\right)$ (since $\left.f_{j} \in A_{\alpha, \epsilon}\right)$, yields

$$
h_{\alpha}\left(f_{0}, f_{n, j}\right)<1-\alpha \delta_{1}-\frac{\epsilon}{2} .
$$

Hence, from (B. (3.), we get

$$
\mathrm{E}\left(L_{n, j}^{\alpha}\right)<\left(1-\alpha \delta_{1}-\frac{\epsilon}{2}\right)^{n} \Pi\left(A_{j}\right)^{\alpha}<\mathrm{e}^{-n\left(\alpha \delta_{1}+\epsilon / 2\right)} \Pi\left(A_{j}\right)^{\alpha} .
$$

As for the numerator of ( $\overline{B .8})$, by the Markov Inequality,

$$
\mathrm{P}\left(\sum_{j \geq 1} L_{n j}^{\alpha}>\mathrm{e}^{n d} \mathrm{e}^{-n\left(\alpha \delta_{1}+\epsilon / 2\right)}\right)<\mathrm{e}^{-n d} \sum_{j \geq 1} \Pi\left(A_{j}\right)^{\alpha} .
$$

Since (B.7) implies that $\sum_{j \geq 1} \Pi\left(A_{j}\right)^{\alpha}<\infty$, we get that $\sum_{j \geq 1} L_{n j}^{\alpha}<\mathrm{e}^{-n\left(\alpha \delta_{1}+\epsilon / 2-d\right)}$ $F_{0}^{\infty}$-a.s. for all large $n$, for any $d>0$. As for the denominator of (B.8), note that ([.6) implies that, for all large $n$ and for any $c>0, I_{n}^{\alpha} \geq \mathrm{e}^{-n \alpha\left(\delta_{1}+c\right)} F_{0}^{\infty}$-a.s.. Therefore,

$$
\Pi_{n}\left(A_{\alpha, \epsilon}\right) \leq \sum_{j \geq 1} \frac{L_{n j}^{\alpha}}{I_{n}^{\alpha}} \leq \mathrm{e}^{-n(\epsilon / 2-d-\alpha c)} \rightarrow 0 \quad F_{0}^{\infty}-\text { a.s. }
$$

by taking $c$ and $d$ sufficiently small.

A corollary to Theorem 1 and Lemma 2 provides the sufficient condition for accumulation of the posterior at $\mathcal{F}_{1}$ in the Hellinger sense.

Corollary 1. Suppose $\Pi$ satisfies (B.5) and that

$$
\sum_{j \geq 1} \Pi\left(B_{j, \varepsilon_{\alpha}}\right)^{\alpha}<\infty
$$

for all $\alpha \in(0,1 / 2)$, where $\varepsilon_{\alpha}=2\left(\alpha^{2} / 2\right)^{1 /(2 \alpha)}$. Then, as $n \rightarrow \infty$, if $\mathcal{F}_{1}$ is nonempty, $\Pi_{n}\left(\left\{f: H\left(\mathcal{F}_{1}, f\right)>\epsilon\right\}\right) \rightarrow 0 F_{0}^{\infty}$-a.s. for any $\epsilon>0$. 
Proof. Condition (B.TU) implies that, for all $\alpha \in(0,1 / 2), \Pi_{n}\left(A_{\alpha}\right) \rightarrow 0 F_{0}^{\infty}$-a.s.. So, clearly,

$$
\Pi_{n}\left(f \in \mathcal{F}: H\left(\overline{A_{\alpha}^{c}}, f\right) \leq \xi\right) \rightarrow 1 \quad F_{0}^{\infty}-\text { a.s. }
$$

for any $\xi>0$ and all $\alpha \in(0,1 / 2)$. Now define $A=\bigcap_{\alpha} \overline{A_{\alpha}^{c}}$ and use (‥D) to get $H(A, f) \leq H\left(\overline{A_{\alpha}^{c}}, f\right)+H\left(\overline{A_{\alpha}^{c}}, A\right)$. Since $A_{\alpha}^{c} \rightarrow A$ in Kuratowski sense, and because of the equivalence of Kuratowski and Hausdorff convergence, $H\left(\overline{A_{\alpha}^{c}}, A\right) \rightarrow 0$ as $\alpha \rightarrow 0$. It follows that, for any $\epsilon>0$, there are $\xi$ and $\alpha$ sufficiently small such that $\{f \in \mathcal{F}: H(A, f) \leq \epsilon\} \subseteq\left\{f \in \mathcal{F}: H\left(\overline{A_{\alpha}^{c}}, f\right) \leq \xi\right\}$, and we can conclude that

$$
\Pi_{n}(f \in \mathcal{F}: H(A, f) \leq \epsilon) \rightarrow 1 \quad F_{0}^{\infty}-\text { a.s. }
$$

for any $\epsilon>0$. The thesis follows from Lemma 2, since $A \subseteq \mathcal{F}_{1}$.

Remark 1. We discuss the alteration of Corollary 1 if $\mathcal{F}_{1}$ is empty. Suppose $\Pi$ satisfies (B.5) and (B.Tl) for all $\alpha \in(0,1 / 2)$, where $\varepsilon_{\alpha}=2\left(\alpha^{2} / 2\right)^{1 /(2 \alpha)}$. Then, as $n \rightarrow \infty$, the posterior still accumulates at $A_{\alpha}^{c}$ for any $\alpha>0$. The lack of elements in $\mathcal{F}_{1}$ now means there is no further development possible. However, it is to be noted that (B.T) is the key condition we need from the prior in order to establish what happens to the posterior no matter the state of $\mathcal{F}_{1}$. Hence, it is this condition we examine in the examples of Section 4.

At this point it is useful to see how consistency is recovered in the wellspecified case. First note that (3.4) for $\alpha=1 / 2$ and any $\epsilon>0$ corresponds to strong consistency in the well-specified case. In fact it is easy to check that the conditions of Theorem 1 for $\delta_{1}=0$ and $\alpha=1 / 2$ correspond to Theorem 4 of Walker (2004). However, as noted by Walker, Lijoi, and Prünster (2005), the prior summability condition can be replaced with an arbitrary power $\alpha$.

Theorem 2 (Walker (20104)). Let $\delta_{1}=0$. If $\Pi$ satisfies (B.5) and (B.7) for some $\alpha \in(0,1)$, then $\Pi_{n}\left(\left\{f: H\left(f_{0}, f\right)>\varepsilon\right\}\right) \rightarrow 0 F_{0}^{\infty}-$ a.s..

For completeness, the proof of Theorem 2 is provided in the Appendix. It is now clear how the sufficient condition in the misspecified case differs from that in the well-specified case: for the latter, according to Theorem 2, condition (3.7) needs to be satisfied for a single $\alpha \in(0,1)$ (and any $\varepsilon>0$ ) in order to have Hellinger consistency.

\section{Examples}

In this section we consider a number of examples. In each case we consider an infinite-dimensional model and find the prior summability conditions in Section 3 established on each prior $\Pi$. If $\Pi$ has full support and inference is possible for the infinite-dimensional model, then we revert to the well-specified case and the 
required condition is weaker ((B.7) only needs to hold for a single $\alpha$ ). However, typically infinite-dimensional models are truncated, or do not have full support, and if $f_{0}$ is out of the range of the truncation or the support of the prior, then our results are required for all $\alpha,(\mathbf{B . 0})$. Specifically, truncation has to be intended in terms of: the number of components in the mixture of priors of Section 4.1; the number of elements in the orthonormal basis of the infinite-dimensional exponential family example of Section 4.2; the support of the prior for the scale parameters $\sigma$ and $\lambda$ in Section 4.3 and 4.4, respectively. We find the condition on the infinite-dimensional model as this obviously covers all levels of truncation.

\subsection{Mixture of priors}

Consider the prior on $\Omega$ given by

$$
\Pi=\sum_{N \geq 1} p_{N} \Pi_{N}
$$

where $\sum_{N \geq 1} p_{N}=1$ and $\Pi_{N}$ is supported on a set of densities $C_{N} \subset \Omega$. This example has been considered in Walker (2004). Let $C_{N} \subseteq C_{N+1}, C_{N}$ increasing to some $\mathcal{F} \subseteq \Omega$ as $N \rightarrow \infty$. We assume that, for each $N, C_{N}$ is totally bounded with respect to the Hellinger metric, $N\left(\epsilon, C_{N}, H\right)<\infty$ for any $\epsilon>0$. An example is given by the Bernstein polynomial prior of Petrone and Wasserman (2002).

For fixed $\epsilon$, let $\left(B_{j, \epsilon}\right)$ be the Hellinger balls of size $\epsilon$ that cover $\mathcal{F}$. We may assume without loss of generality that, for $I_{N}:=N\left(\epsilon, C_{N}, H\right), C_{N} \subseteq \bigcup_{j \leq I_{N}} B_{j, \epsilon}$, so that $\Pi_{N}\left(B_{j, \epsilon}\right)=0$ for any $j>I_{N}$. For $\alpha \in(0,1)$, we consider

$$
\sum_{j \geq 1} \Pi\left(B_{j, \epsilon}\right)^{\alpha}=\sum_{j \geq 1}\left(\sum_{N: I_{N} \geq j} p_{N} \Pi_{N}\left(B_{j, \epsilon}\right)\right)^{\alpha} \leq \sum_{j \geq 1}\left(\sum_{N \geq M_{j}} p_{N}\right)^{\alpha},
$$

where $M_{j}=\min \left\{N: I_{N} \geq j\right\}$. Since $I_{N}$ depends on $\epsilon$, so does $M_{j}$, hence we write $M_{j}(\epsilon)$. Consequently, by defining $\bar{P}(m)=\sum_{N \geq m} p_{N}$, if $\sum_{j \geq 1} \bar{P}\left(M_{j}(\epsilon)\right)^{\alpha}<\infty$, then (B.]) holds. Hence, it is sufficient that

$$
\bar{P}\left(M_{j}(\epsilon)\right)<a j^{-(1 / \alpha)-r}
$$

for some $r>0$ and $a>0$ for all large $j$.

For example if $I_{N}=(c / \epsilon)^{N}$, for some $c$ not depending on $\epsilon$, as in the case of Bernstein polynomial prior, then $M_{j}(\epsilon)=\lfloor\log j / \log (c / \epsilon)\rfloor$, so that (4.]) yields

$$
\bar{P}(N)<a\left(\frac{c}{\epsilon}\right)^{-((1 / \alpha)+r) N}=a \exp \{-\psi(\epsilon, \alpha) N\},
$$

where $\psi(\epsilon, \alpha)=\log (c / \epsilon)\left(\alpha^{-1}+r\right)$. Note that (4.2) puts a constraint on the prior mass on large $C_{N}$. Since $\psi(\epsilon, \alpha)$ increases to $\infty$ as $\epsilon$ decreases to zero, (B.7) holds 
for any $\epsilon>0$ (consistency in the well-specified case) if $\bar{P}(N)<a \mathrm{e}^{-\psi N}$ for any $\psi>0$ and all large $N$, which holds if $N^{-1} \log \bar{P}(N) \rightarrow-\infty$. Hence, we recover the condition of Section 6 in Walker (2004). Establishing (‥10) does not change the result, in fact $\psi\left(\varepsilon_{\alpha}, \alpha\right)$ also increases to $\infty$ as $\alpha$ decreases to zero. Therefore, in this example, the required condition for the misspecified case is that for the well-specified case.

\subsection{Infinite-dimensional exponential family}

Let $\Theta=\left(\theta_{j}\right)_{j \geq 1}$ be a sequence of independent random variables with $\theta_{j} \sim$ $N\left(0, \sigma_{j}^{2}\right)$, and let $\left(\phi_{j}\right)_{j \geq 1}$ be a sequence of orthogonal polynomials on [0,1]. Define the family of densities as

$$
f_{\Theta}(x)=\exp \left\{\sum_{j \geq 1} \theta_{j} \phi_{j}(x)-c(\Theta)\right\},
$$

where $c(\Theta)$ makes $f_{\Theta}$ a density. This example has been considered in Barron, Schervish, and Wasserman (1199.9) and Walker (20104). For illustration, we work with the orthonormal basis

$$
\phi_{1}(x)=1 \quad \text { and } \phi_{j}(x)=\sqrt{2} \cos (j \pi x) \text { for } j \geq 2,
$$

so that $\left\|\phi_{j}\right\|_{\infty}=\sqrt{2}$ and $\left\|\phi_{j}^{\prime}\right\|_{\infty}=j$, for any $j \geq 2$. To ensure that $f_{\Theta}$ is a density with probability 1 , it is sufficient that $\sum_{j} \sigma_{j}<\infty$.

We next consider how to construct a Hellinger covering $\left(B_{j, \epsilon}\right)_{j \geq 1}$ in (B.7) for the density set $\mathcal{F}$ on which $\Pi$ is supported, the prior being induced by the distribution on the infinite sequence $\Theta$. Suppose, for $i=1,2$, we put

$$
f_{i}(x)=\frac{\mathrm{e}^{w_{i}(x)}}{\int \mathrm{e}^{w_{i}(y)} \mathrm{d} y} .
$$

Then, $\left\|w_{1}-w_{2}\right\|_{\infty} \leq \epsilon$ implies $H\left(f_{1}, f_{2}\right) \leq \epsilon \mathrm{e}^{\epsilon / 2}$ (see Lemma 3.1 in van der Vaart and van Zanten (201)8) ). Now take $f_{1}=f_{\Theta_{1}}$ and $f_{2}=f_{\Theta_{2}}$ for sequences $\Theta_{1}=\left(\theta_{1 j}\right)$ and $\Theta_{2}=\left(\theta_{2 j}\right)$ such that $w_{i}(x)=\sum_{j} \theta_{i j} \phi_{j}(x)$. Also, take $\Theta_{1}$ and $\Theta_{2}$ close in the sense that

$$
\left|\theta_{1 j}-\theta_{2 j}\right|<\delta_{j}=\frac{\delta \omega_{j}}{\sum_{j \geq 1} \omega_{j}}
$$

for some sequence $\left(\omega_{j}\right)$ satisfying $\sum_{j} \omega_{j}<\infty$. Then

$$
\left\|\sum_{j \geq 1} \theta_{1 j} \phi_{j}-\sum_{j \geq 1} \theta_{2 j} \phi_{j}\right\|_{\infty}=\sqrt{2} \delta
$$

and $H\left(f_{\Theta_{1}}, f_{\Theta_{2}}\right) \leq \sqrt{2} \delta \mathrm{e}^{\delta / \sqrt{2}}$. It follows that $B_{j, \epsilon}$ can be taken as set of the type

$$
\left\{f_{\Theta}: n_{j} \delta_{j}<\theta_{j}<\left(n_{j}+1\right) \delta_{j}\right\}
$$


for $\delta_{j}=\delta(\epsilon) \omega_{j} / \sum_{j \geq 1} \omega_{j}$ with $\delta(\epsilon)=g^{-1}(\epsilon) / \sqrt{2}, g^{-1}$ being the inverse of $g(x)=$ $x \mathrm{e}^{x / 2}$, and integers $\left(n_{j}\right)$ that vary between $-\infty$ and $+\infty$. Note that $\delta(\epsilon)$ is monotonic increasing in $\epsilon$, with $\delta(0)=0$. Since $\theta_{j} \sim N\left(0, \sigma_{j}^{2}\right)$, with independence across $j$, in view of (B.7), we are interested in the finiteness of

$$
\sum_{n_{1}=-\infty}^{\infty} \ldots \sum_{n_{M}=-\infty}^{\infty} \prod_{j=1}^{\infty}\left(\operatorname{Pr}\left[n_{j} \delta_{j}<\theta_{j}<\left(n_{j}+1\right) \delta_{j}\right]\right)^{\alpha} .
$$

Due to symmetry, this holds if

$$
\prod_{j=1}^{\infty} \sum_{n=0}^{\infty}\left(\operatorname{Pr}\left[n \delta_{j}<\theta_{j}<(n+1) \delta_{j}\right]\right)^{\alpha}
$$

Next, we have

$$
\begin{aligned}
\sum_{n=0}^{\infty}\left(\operatorname{Pr}\left[n \delta_{j}<\theta_{j}<(n+1) \delta_{j}\right]\right)^{\alpha} & \leq 1+(2 \pi)^{-\alpha / 2}\left(\frac{\delta_{j}}{\sigma_{j}}\right)^{\alpha} \sum_{n=1}^{\infty} \exp \left\{-\frac{\alpha \delta_{j}^{2} n}{2 \sigma_{j}^{2}}\right\} \\
& \leq 1+(2 \pi)^{-\alpha / 2}\left(\frac{\delta_{j}}{\sigma_{j}}\right)^{\alpha}\left[\exp \left\{\frac{\alpha \delta_{j}^{2}}{2 \sigma_{j}^{2}}\right\}-1\right]^{-1} .
\end{aligned}
$$

Note that, for any $m \geq 1, \mathrm{e}^{z}-1 \geq z^{m} / m$ !, so that we can use the inequality

$$
\left[\exp \left\{\frac{\alpha \delta_{j}^{2}}{2 \sigma_{j}^{2}}\right\}-1\right]^{-1} \leq \frac{m !\left(2 \sigma_{j}^{2}\right)^{m}}{\alpha^{m} \delta_{j}^{2 m}}
$$

to get

$$
\begin{aligned}
\sum_{n=0}^{\infty}\left(\operatorname{Pr}\left[n \delta_{j}<\theta_{j}<(n+1) \delta_{j}\right]\right)^{\alpha} & \leq 1+(2 \pi)^{-\alpha / 2}\left(\frac{\delta_{j}}{\sigma_{j}}\right)^{\alpha} \frac{m !\left(2 \sigma_{j}^{2}\right)^{m}}{\alpha^{m} \delta_{j}^{2 m}} \\
& =1+\left(\frac{2}{\alpha}\right)^{m} m !(2 \pi)^{-\alpha / 2}\left(\frac{\sigma_{j}}{\delta_{j}}\right)^{2 m-\alpha} .
\end{aligned}
$$

Substituting for $\delta_{j}=\delta(\epsilon) \omega_{j} / \sum_{j} \omega_{j}$, we get

$$
\sum_{n=0}^{\infty}\left(\operatorname{Pr}\left[n \delta_{j}<\theta_{j}<(n+1) \delta_{j}\right]\right)^{\alpha} \leq 1+\psi(\epsilon, \alpha)\left(\frac{\sigma_{j}}{\omega_{j}}\right)^{2 m-\alpha}
$$

where

$$
\psi(\epsilon, \alpha)=\left(\frac{2}{\alpha}\right)^{m} m !(2 \pi)^{-\alpha / 2}\left(\frac{\delta(\epsilon)}{\sum_{j \geq 1} \omega_{j}}\right)^{2 m-\alpha} .
$$


The required condition on the $\left(\sigma_{j}\right)$ is then that

$$
\prod_{j=1}^{\infty}\left\{1+\psi(\epsilon, \alpha)\left(\frac{\sigma_{j}}{\omega_{j}}\right)^{2 m-\alpha}\right\}<\infty,
$$

or that $\sum_{j=1}^{\infty} \log \left\{1+\psi(\epsilon, \alpha)\left(\sigma_{j} / \omega_{j}\right)^{2 m-\alpha}\right\}<\infty$, which holds if

$$
\psi(\epsilon, \alpha) \sum_{j=1}^{\infty}\left(\frac{\sigma_{j}}{\omega_{j}}\right)^{2 m-\alpha}<\infty .
$$

The convergence of this series requires a restriction on how the sequence $\left(\sigma_{j}\right)$ grows as $j \rightarrow \infty$. Moreover, we see that the size $\epsilon$ of the Hellinger covering does not play any role. Now, if we put $\omega_{j} \propto j^{-1-r}$ for any $r>0$, then the condition

$$
\sum_{j=1}^{\infty}\left(\sigma_{j} j^{1+r}\right)^{2 m-\alpha}<\infty
$$

is sufficient. Therefore, we can actually have $\sigma_{j} \propto j^{-1-q}$ for any $q>0$, by choosing $r<q$ and $m$ large enough such that $(r-q) 2 m$ is sufficiently smaller than -1 . We also see that $\alpha$ does not affect condition (4.3), therefore $\sigma_{j} \propto j^{-1-q}$ for any $q>0$ is sufficient for ([.]) to hold for any $\epsilon>0$ and for (3.10) to hold for any $\alpha$ sufficiently small. This means that the condition in Section 6 of Walker (20104) for consistency works also in the misspecified case.

\subsection{Mixtures of normal densities}

We consider priors obtained via a nonparametric mixture of normal densities; see Ghosal, Ghosh, and Ramamoorthi (11999) and Lijoi, Prünster, and Walker (2010.5). Let $\widetilde{P}$ be a discrete random probability distribution on $\mathbb{R}$ with law $\Lambda$ and prior guess $P_{0}$. For $\phi_{\sigma}$, the density function of the normal with mean 0 and variance $\sigma^{2}$, we model the density as

$$
\tilde{f}_{\sigma, \widetilde{P}}(x)=\phi_{\sigma} * \widetilde{P}=\int \phi_{\sigma}(x-\theta) \widetilde{P}(\mathrm{~d} \theta)
$$

and $\sigma$, with prior distribution $\mu$, is supported on the interval $[0, \bar{\sigma}]$.

We follow the proof of Theorem 1 in Lijoi, Prünster, and Walker (2010.5) by defining the sets

$$
\mathscr{F}_{\underline{\sigma}, a, \delta}^{\bar{\sigma}}=\bigcup_{\underline{\sigma}<\sigma<\bar{\sigma}}\left\{\phi_{\sigma} * P: P([-a, a]) \geq 1-\delta\right\},
$$

where $\underline{\sigma}, a>0$. Recall that $H^{2}(f, g) \leq\|f-g\|_{1}$, where $\|f-g\|_{1}=\int|f-g|$ is the $L_{1}$-distance between $f$ and $g$. Hence we have $N(\sqrt{\delta}, \mathscr{G}, H) \leq N\left(\delta, \mathscr{G},\|\cdot\|_{1}\right)$. 
From Ghosal, Ghosh, and Ramamoorthi ([1999), the upper bound for the $L_{1^{-}}$ metric entropy of set $\mathscr{F}_{\underline{\sigma}, a, \delta}^{\bar{\sigma}}$ is given by

$$
\log N\left(\delta, \underset{\mathscr{F}^{\alpha}, a, \delta}{\bar{\sigma}},\|\cdot\|_{1}\right) \leq C_{\delta} \frac{a}{\underline{\sigma}}
$$

where $C_{\delta}=K \delta^{-1} \log (1 / \delta)$ for some constant $K$. Hence, $\log N\left(\delta, \mathscr{F}_{\underline{\sigma}, a, \delta}^{\bar{\sigma}}, H\right) \leq$ $C_{\delta^{2}} a / \underline{\sigma}$. Now let $\left(a_{n}\right)_{n \geq 1}$ be an increasing sequence of positive numbers such that $a_{0}=0$ and $\lim _{n} a_{n}=\infty$, and let $\left(\sigma_{n}\right)_{n \geq 1}$ be a decreasing sequence of positive numbers such that $\sigma_{0}=\bar{\sigma}$ and $\lim _{n} \sigma_{n}=0$. Set

$$
\mathscr{G}_{\sigma_{k}, a_{j}, \delta^{2}}^{\bar{\sigma}}=\bigcup_{\sigma_{k}<\sigma<\bar{\sigma}}\left\{\phi_{\sigma} * P: P\left(\left[-a_{j}, a_{j}\right]\right) \geq 1-\delta^{2}, P\left(\left[-a_{j-1}, a_{j-1}\right]\right)<1-\delta^{2}\right\},
$$

so that $\bigcup_{j, k} \mathscr{G}_{\sigma_{k}, a_{j}, \delta^{2}}^{\bar{\sigma}}=\mathcal{F}$. Reasoning as in Lijoi, Prünster, and Walker (201015), for any $\delta$ there is an integer $N$ such that $\mathscr{G}_{\sigma_{k}, a_{j}, \delta^{2}}^{\bar{\sigma}}$ is included in $\mathscr{F}_{\sigma_{k}, a_{N}, \delta^{2}}^{\bar{\sigma}}$, so

$$
\log N\left(\delta, \mathscr{G}_{\sigma_{k}, a_{j}, \delta^{2}}^{\bar{\sigma}}, H\right) \leq C_{\delta^{2}} \frac{a_{N}}{\sigma_{k}} .
$$

This means that for each $j$ and $k, \mathscr{G}_{\sigma_{k}, a_{j}, \epsilon^{2}}^{\bar{a}}$ has a finite Hellinger $\epsilon$-covering $\left\{B_{j k l, \epsilon}\right.$ : $\left.l=1, \ldots, N_{j, k}\right\}$, where $N_{j, k} \leq \exp \left\{C_{\epsilon^{2}} a_{N} / \sigma_{k}\right\}$, so that we consider $\left(B_{j k l, \epsilon}\right)$ in establishing ([3.7). Now, for each $j \geq 1$, define the sets

$$
B_{j, \epsilon}^{\prime}=\left\{P: P\left(\left[-a_{j}, a_{j}\right]\right) \geq 1-\epsilon^{2}, P\left(\left[-a_{j-1}, a_{j-1}\right]\right)<1-\epsilon^{2}\right\} .
$$

The condition (3.7) is implied by the finiteness of the sum

$$
\begin{aligned}
\sum_{j, k \geq 1} \sum_{l=1}^{N_{j, k}} \Pi\left(B_{j k l, \epsilon}\right)^{\alpha} & \leq \sum_{j, k \geq 1} N_{j, k}^{1-\alpha} \Pi\left(\mathscr{G}_{\sigma_{k}, a_{j}, \epsilon^{2}}^{\bar{\sigma}}\right)^{\alpha} \\
& \leq \sum_{k \geq 1} \mathrm{e}^{(1-\alpha) C_{\epsilon^{2}} a_{N} / \sigma_{k}} \mu\left(\sigma_{k}<\sigma \leq \sigma_{k-1}\right)^{\alpha} \sum_{j \geq 1} \Lambda\left(B_{j, \epsilon}^{\prime}\right)^{\alpha}
\end{aligned}
$$

where the inequality (4.4) follows by the monotonicity of power means. We deal with the inner sum first, showing that $\sum_{j \geq 1} \Lambda\left(B_{j, \epsilon}^{\prime}\right)^{\alpha}<\infty$ for any $\epsilon>0$ and any $\alpha \in(0,1)$ is implied by

$$
P_{0}\left([-a, a]^{c}\right) \leq \mathrm{e}^{-\eta a}
$$

for some $\eta>0$ and $a$ sufficiently large. In fact, since $B_{j, \epsilon}^{\prime} \subset\left\{P: P\left(\left[-a_{j-1}, a_{j-1}\right]^{c}\right)\right.$ $\left.>\epsilon^{2}\right\}$, an application of Markov Inequality, together with (4.5), yields

$$
\sum_{j \geq 1} \Lambda\left(B_{j, \epsilon}^{\prime}\right)^{\alpha} \leq \epsilon^{-2 \alpha} \sum_{j \geq 1} P_{0}\left(\left[-a_{j-1}, a_{j-1}\right]^{c}\right)^{\alpha} \leq \epsilon^{-2 \alpha} \sum_{j \geq 1} \mathrm{e}^{-\alpha \eta a_{j-1}} .
$$


Then, by taking $a_{j} \sim j$ as $j \rightarrow \infty, a_{j}>(\alpha \eta)^{-1}(1+s) \log j$ for $j$ sufficiently large and some $s>0$, so that $\sum_{j \geq 1} \mathrm{e}^{-\alpha \eta a_{j-1}}<\infty$ for any $\alpha$ arbitrarily small (the size of $\epsilon$ does not play any role). Note that (4.5) is stronger than the tail condition on $P_{0}$ needed for consistency, $\int|\theta| P_{0}(\mathrm{~d} \theta)<\infty$, see Theorem 1 in Lijoi, Prünster, and Walker (2005). In fact, the latter only implies $P_{0}\left([-a, a]^{c}\right)=O\left(a^{-(1+r)}\right)$ for $r>0$, and so we get the convergence of the series $\sum_{j \geq 1} \Lambda\left(B_{j, \epsilon}\right)^{\alpha}$ only for $\alpha>(1+r)^{-1}$. At this stage we are left to establish that

$$
\sum_{k \geq 1} \mathrm{e}^{(1-\alpha) C_{\epsilon^{2} a_{N} / \sigma_{k}}} \mu\left(\sigma_{k}<\sigma \leq \sigma_{k-1}\right)^{\alpha}<\infty .
$$

If we assume that

$$
\mu\left\{\sigma<k^{-1}\right\} \leq \mathrm{e}^{-\gamma k}
$$

then

$$
\sum_{k \geq 1} \mathrm{e}^{(1-\alpha) C_{\epsilon^{2}} a_{N} / \sigma_{k}} \mu\left(\sigma_{k}<\sigma \leq \sigma_{k-1}\right)^{\alpha} \leq \sum_{k \geq 1} \mathrm{e}^{-\left(\gamma \alpha-(1-\alpha) C_{\left.\epsilon^{2} a_{N}\right) / \sigma_{k}}\right.}
$$

Let $\psi(\epsilon, \alpha)=\alpha^{-1}(1-\alpha) C_{\epsilon^{2}} a_{N}$, which goes to infinity as either $\alpha$ or $\epsilon$ go to zero. Now set $\sigma_{k}<\psi(\epsilon, \alpha)(1-s)^{-1} / \log k$ for $k$ sufficiently large and for some $s>0$; this is possible for any $\alpha$ and $\epsilon$ by taking, for example, $\sigma_{k} \sim 1 / k$ as $k \rightarrow \infty$. Then the finiteness of the series in the r.h.s. of the last display is implied by $\gamma>\psi(\epsilon, \alpha)$ in (4.6), so that in the well-specified case and the misspecified case we need (4.6) to hold for any $\gamma>0$.

\subsection{Gaussian process priors}

We consider random densities obtained as logistic transformations of Gaussian processes; see Leonard (1.978), Lenk (1.988, 1.991), Tokdar and Ghosh (2007), and van der vaart and van Zanten (2008). Let $I$ be a fixed bounded interval $I$ in $\mathbb{R}$, and let

$$
f(x)=\frac{\mathrm{e}^{W(x)}}{\int_{I} \mathrm{e}^{W(s)} \mathrm{d} s},
$$

where $\{W(x), x \in I\}$ is a Gaussian process with mean function $\mu(t)$ and covariance kernel $\sigma(s, t)=\operatorname{Cov}(W(s), W(t))$. Without loss of generality we take $\mu=0$ and $I=(0,1)$. Moreover, we let $\sigma$ depend on a parameter $\lambda>0$ via

$$
\sigma(s, t)=\sigma_{0}(\lambda s, \lambda t)
$$

where $\sigma_{0}$ is a fixed covariance kernel and $\lambda$ has prior distribution $\Pi_{\lambda}$, supported on $\mathbb{R}_{+}$. Let $W_{0}$ be the Gaussian process with covariance $\sigma_{0}$ so that $W_{0}(\lambda t)$ has 
covariance $\sigma_{0}(\lambda s, \lambda t)$. Then, the random density $f$ is modeled by

$$
\begin{aligned}
f \mid W, \lambda & =\frac{\mathrm{e}^{W(x)}}{\int_{I} \mathrm{e}^{W(s)} \mathrm{d} s} \\
W \mid \lambda & =W_{0}(\lambda \cdot)
\end{aligned}
$$

with $\lambda$ and $W_{0}$ independent. This defines a prior distribution $\Pi$ on $\Omega$, the space of densities on $I$. As before, $\mathcal{F}$ is the support of $\Pi$.

The Kullback-Leibler support of $\Pi$ has been studied in Tokdar and Ghosh (2007), see also Ghosal and Roy (2006). With a regularity condition on $\sigma_{0}$, which we assume to be satisfied (see, e.g., Theorem 5 in Ghosal and Roy (2006)), $W_{0}(\cdot)$ has differentiable sample paths and the derivative process $D W_{0}(\cdot)$ is Gaussian with continuous sample paths. If we take

$$
\sigma_{1}(s, t)=\frac{\partial^{2}}{\partial s \partial t} \sigma_{0}(s, t),
$$

to be the covariance kernel of $D W_{0}(t)$, then the derivative process $D W_{0}(\cdot)$ is sub-Gaussian with respect to the Euclidean distance

$$
\mathrm{E}\left(D W_{0}(s)-D W_{0}(t)\right)^{2} \leq c_{1}(s-t)^{2}
$$

for some constant $c_{1}$ depending on $\sigma_{1}$. Define $\sigma_{1}^{2}\left(W_{0}\right)=\sup _{t \in I} \operatorname{Var}\left(D W_{0}(t)\right)<$ $\infty$. Then, an application of Proposition A.2.7 of van der Vaart and Wellner (1996), yields

$$
\Pi\left(\sup _{t \in I}|D W(t)|>a \mid \lambda\right) \leq c_{2} \exp \left\{-c_{3} a^{2} \lambda^{-2}\right\}
$$

for some positive constants $c_{2}$ and $c_{3}$. Finally, Theorem 2.7.1 of van der Vaart and Wellner (19966) gives the entropy bound

$$
\log N\left(\epsilon,\left\{w: \sup _{t \in I}|D w(t)| \leq a\right\},\|\cdot\|_{\infty}\right) \leq c_{4} \frac{a}{\epsilon}
$$

for some positive constant $c_{4}$. Let $\left(a_{n}\right)_{n \geq 1}$ be an increasing sequence of positive real numbers such that $a_{0}=0$ and $\lim _{n} a_{n}=\infty$. For

$$
B_{j}=\left\{w: a_{j-1}<\sup _{t \in I}|D w(t)|<a_{j}\right\}
$$

define $F_{j}=\left\{f(x)=\mathrm{e}^{w(x)} / \int_{I} \mathrm{e}^{w(s)} \mathrm{d} s: w \in B_{j}\right\}$. Then the sets $\left(F_{j}\right)_{j \geq 1}$ are pairwise disjoint and form a partition of $\mathcal{F}$. Since $N\left(\epsilon \mathrm{e}^{\epsilon}, F_{j}, H\right) \leq N\left(\epsilon, B_{j},\|\cdot\|_{\infty}\right)$, see Section 4.2 , there is no loss of generality in working with the sets $B_{j}$ and the sup norm. 
From (4.8), each $B_{j}$ has a finite $\epsilon$-covering $\left\{C_{j l, \epsilon}: l=1,2, \ldots, N_{j}\right\}$, where $N_{j} \leq \exp \left(c_{4} a_{j} / \epsilon\right)$. Hence we consider $\left(C_{j l, \epsilon}\right)$ in establishing (B.]), that is, we are interested in the finiteness of the sum

$$
\sum_{j \geq 1} \sum_{l=1}^{N_{j}} \Pi\left(C_{j l}\right)^{\alpha} \leq \sum_{j \geq 1} N_{j}^{1-\alpha} \Pi\left(B_{j}\right)^{\alpha},
$$

where we have used the monotonicity of power means as in (4.4). Since $N_{j}^{1-\alpha}$ grows exponentially in $a_{j}$, we need to control the behavior of $\Pi\left(B_{j}\right)$ as $j$ goes to infinity. Now, from (4.7),

$$
\begin{aligned}
\Pi\left(B_{j}\right) & \leq \int_{0}^{\infty} \Pi\left(\sup _{t \in I}|D W(t)|>a_{j-1} \mid \lambda\right) \Pi_{\lambda}(\mathrm{d} \lambda) \\
& \leq \int_{0}^{\infty} c_{2} \exp \left\{-c_{3} a_{j-1}^{2} \lambda^{-2}\right\} \Pi_{\lambda}(\mathrm{d} \lambda),
\end{aligned}
$$

and therefore we need to study the behavior, as $a_{j} \rightarrow \infty$, of a Laplace-type transform of the prior $\Pi_{\lambda}$. To this end, we resort to a suitable version of the Tauberian Theorem to show that if

$$
\Pi_{\lambda}(\lambda>t) \sim \mathrm{e}^{-\gamma t^{2}}
$$

for some $\gamma>0$ and $t$ sufficiently large, then there exists a positive constant $c_{5}$ such that

$$
\Pi\left(B_{j}\right) \leq \mathrm{e}^{-c_{5} \gamma a_{j-1}}
$$

as $j \rightarrow \infty$. The result follows by an application of Theorem 4.12.9 of Bingham, Goldie, and Teugels (1987). Let $\mu$ be a measure on $(0, \infty)$ whose Laplace transform $M(\tau)=\int_{0}^{\infty} \mathrm{e}^{-\tau x} \mathrm{~d} \mu(x)$ converges for all $\tau>0$. With their notation, we choose $\alpha=-1$ and $\phi(\tau) \sim \tau^{-1}$ as $\tau \rightarrow 0^{+}$. Then, for $B>0$,

$$
-\log \mu(0, x] \sim B x^{-1} \quad\left(x \rightarrow 0^{+}\right) \quad \text { iff } \quad-\log M(\tau) \sim 2 B^{1 / 2} \tau^{1 / 2} \quad(\lambda \rightarrow \infty) .
$$

Now, with $y=1 / \sqrt{x}$, write $M(\tau)=\int_{0}^{\infty} \mathrm{e}^{-\tau y^{-2}} \mathrm{~d} \eta(y)$ for the measure $\eta$ on $(0, \infty)$ defined as $\eta(0, y]=\mu\left[y^{-2}, \infty\right)$. Then

$$
-\log \eta(y, \infty) \sim B y^{2} \quad(y \rightarrow \infty) \quad \text { iff } \quad-\log M(\tau) \sim 2 B^{1 / 2} \tau^{1 / 2} \quad(\lambda \rightarrow \infty) .
$$

Put $\eta(y, \infty)=\Pi_{\lambda}(y, \infty)$ in (4.22) and assume that (4.10) is in force. Also put $B=\gamma$ and $\tau=c_{3} a_{j-1}^{2}$ in (4.12). Then

$$
M\left(c_{3} a_{j-1}^{2}\right)=\int_{0}^{\infty} \mathrm{e}^{-c_{3} a_{j-1}^{2} \lambda^{-2}} \Pi_{\lambda}(\mathrm{d} \lambda) \sim \exp \left\{-2 \gamma^{1 / 2} c_{3}^{1 / 2} a_{j-1}\right\}
$$


as $j \rightarrow \infty$. It is now easy to see that (4.T0) implies (4.T]). Back to (4.9), by using (4.8) and (4.Tा) we get

$$
\sum_{j \geq 1} N_{j}^{1-\alpha} \Pi\left(B_{j}\right)^{\alpha} \leq \sum_{j \geq 1} \exp \left\{-\left(\alpha c_{5} \gamma-\frac{c_{4}}{\epsilon}(1-\alpha)\right) a_{j}\right\} .
$$

Thus, under (4.JU), finiteness of the series in (4.9) and, in turn (B.7), is implied by $\gamma>\psi(\alpha, \epsilon)$, where

$$
\psi(\alpha, \epsilon)=\frac{c_{4}(1-\alpha)}{\epsilon \alpha c_{5}} .
$$

Since $\psi(\epsilon, \alpha)$ goes to infinity as either $\alpha$ or $\epsilon$ go to zero, we conclude that the same sufficient condition applies for (B.7) for any $\epsilon>0$ (consistency in the wellspecified case) and for (B.TU) for any $\alpha>0$, namely that (4.10) is satisfied for any $\gamma>0$.

\section{Discussion}

In this paper, we have generalized the condition for consistency in the wellspecified case to asymptotic results in the misspecified case. Illustrations considered suggest that it is not too problematic to implement the sufficient conditions on the prior.

The sufficient conditions we find for the prior allow us to say what happens to the posterior under all scenarios. Basically, whether $\mathcal{F}_{1}$ is empty or not, we establish asymptotics for either case. The search when $\mathcal{F}_{1}$ is finite and nonempty has been important due to the lack of general theory for the case when $\mathcal{F}_{1}$ is empty. To our knowledge we are the first to describe some general theory for the asymptotics when $\mathcal{F}_{1}$ is empty; though we are aware of the special case of the Bernstein polynomial prior studied in Petrone and Wasserman (20102).

In each case of misspecified prior $\Pi$ we would find it difficult to find the $C_{\Pi}$ such that if $f_{0} \in C_{\Pi}$ then $\mathcal{F}_{1}$ is empty, whereas if $f_{0} \in C_{\Pi}^{c}$ then $\mathcal{F}_{1}$ is non-empty. But of course we would also find it difficult to use this information since the location of $f_{0}$ is not known. It would therefore be useful to identify $\Pi$ for which $C_{\Pi}$ is empty, something which has been established only when $\mathcal{F}$ is convex.

\section{Acknowledgement}

The authors would like to thank an associate editor and a referee for important comments and suggestions. Moreover, S. Tokdar is gratefully acknowledged for some useful discussions. This work was supported by the European Research Council (ERC) through StG "N-BNP" 306406. 


\section{Appendix}

Proof of Lemma 1. Start with the inequality

$$
\left|h_{\alpha}\left(f_{0}, f\right)-h_{\alpha}\left(f_{0}, g\right)\right| \leq \int\left|\left(\frac{f}{f_{0}}\right)^{\alpha}-\left(\frac{g}{f_{0}}\right)^{\alpha}\right| f_{0} .
$$

Next, note that, for any $a, b>0$,

$$
\left|a^{\alpha}-b^{\alpha}\right|^{1 / \alpha} \leq\left|a^{\beta}-b^{\beta}\right|^{1 / \beta}, \quad \text { for } 0 \leq \alpha \leq \beta .
$$

To see this, it is sufficient to take $a>b$ and show that $\tau(\alpha)=\left(a^{\alpha}-b^{\alpha}\right)^{1 / \alpha}$ is non decreasing for $\alpha>0$. The latter is equivalent to $\left(\mathrm{e}^{\alpha}-1\right)^{1 / \alpha}$ non decreasing for $\alpha \geq 0$, and, in turn, to $\log (x-1) / \log (x)$ non decreasing for $x>1$. The last statement can be easily checked by looking at the first derivative and using the inequality $(x-1) \log x \leq x \log x$ for $x>1$. Since, by hypothesis, $\alpha \leq 1 / 2$, (A.2) yields an upper bound on the right hand side of (A. 1 ):

$$
\int\left|\left(\frac{f}{f_{0}}\right)^{\alpha}-\left(\frac{g}{f_{0}}\right)^{\alpha}\right| f_{0} \leq \int\left|\left(\frac{f}{f_{0}}\right)^{1 / 2}-\left(\frac{g}{f_{0}}\right)^{1 / 2}\right|^{2 \alpha} f_{0} .
$$

Now use $\mathrm{E}|X|^{p} \leq(\mathrm{E}|X|)^{p}$ for any $0<p<1$ to get

$$
\left|h_{\alpha}\left(f_{0}, f\right)-h_{\alpha}\left(f_{0}, g\right)\right| \leq\left(\int\left|\left(\frac{f}{f_{0}}\right)^{1 / 2}-\left(\frac{g}{f_{0}}\right)^{1 / 2}\right|^{2} f_{0}\right)^{\alpha} .
$$

Finally, note that $\int\left|\left(f / f_{0}\right)^{1 / 2}-\left(g / f_{0}\right)^{1 / 2}\right|^{2} f_{0}=\int\left(f^{1 / 2}-g^{1 / 2}\right)^{2}=H^{2}(f, g)$.

Proof of Lemma 2. Let $\alpha_{m}$ and $\epsilon_{m}$ be two positive sequences decreasing to 0 such that $\epsilon_{m}^{2 \alpha_{m}} / \alpha_{m} \rightarrow 0$. Next, let $f \in \bigcap_{m} \overline{A_{\alpha_{m}}^{c}}$ so that, for each $m$, there exists a sequence $\left(f_{m, r_{m}}\right)_{r_{m}=1}^{\infty} \in \mathcal{F}$ such that

$$
\begin{aligned}
& H\left(f, f_{m, r_{m}}\right) \rightarrow 0 \quad \text { as } r_{m} \rightarrow \infty \\
& d_{\alpha_{m}}\left(f_{0}, f_{m, r_{m}}\right) \leq \delta_{1}+\alpha_{m} \text { for all } r_{m}
\end{aligned}
$$

Clearly, $f \in \overline{\mathcal{F}}$. Moreover, by using Lemma 1 and the identity $d_{\alpha}(f, g)=\alpha^{-1}\{1-$ $\left.h_{\alpha}(f, g)\right\}$, we have

$$
d_{\alpha_{m}}\left(f_{0}, f\right)<d_{\alpha_{m}}\left(f_{0}, f_{m, r}\right)+\frac{1}{\alpha_{m}} H\left(f, f_{m, r_{m}}\right)^{2 \alpha_{m}}
$$

By the hypothesis made, we can take, for each $m, r_{m}$ large enough such that $H\left(f, f_{m, r_{m}}\right) \leq \epsilon_{m}$ to get

$$
d_{\alpha_{m}}\left(f_{0}, f\right)<\delta_{1}+\alpha_{m}+\frac{1}{\alpha_{m}} \epsilon_{m}^{2 \alpha_{m}} .
$$


Since the last inequality holds for any $m$, hence for $\alpha_{m}$ going to 0 , we exploit the convergence of $d_{\alpha_{m}}\left(f_{0}, f\right)$ to $D\left(f_{0}, f\right)$ to conclude that

$$
D\left(f_{0}, f\right)<\delta_{1}+\beta
$$

for any $\beta$ small enough. This implies that $f \in \mathcal{F}_{1}$, completing the proof.

Proof of Theorem 2. When $\alpha \leq 1 / 2, \Pi\left(B_{j, \epsilon}\right)^{1 / 2} \leq \Pi\left(B_{j, \epsilon}\right)^{\alpha}$, so that consistency follows from Theorem 4 in Walker (20104). Let then (3.7) be satisfied for $1 / 2<\alpha<1$. We aim at establishing that $\Pi_{n}\left(A_{1 / 2, \epsilon^{2} / 2}\right) \rightarrow 0 F_{0}^{\infty}$-a.s. as $n \rightarrow \infty$, (ए:) when $\delta_{1}=0$ and (B. $)$. Reasoning as in the proof of Theorem 1, we consider a partition $\left(A_{j}\right)_{j \geq 1}$ of $A_{1 / 2, \epsilon^{2} / 2}$ such that $A_{j} \subseteq A_{j}^{*}=\left\{f: H\left(f_{j}, f\right)<\epsilon / 2\right\}$, $f_{j} \in A_{1 / 2, \epsilon^{2} / 2}$ so that $d_{1 / 2}\left(f_{0}, f_{j}\right)>\epsilon^{2}$. Then, any $f \in A_{j}$ has $d_{1 / 2}\left(f_{0}, f\right)>\epsilon^{2} / 2$ and $h_{1 / 2}\left(f_{0}, f\right)<1-\epsilon^{2} / 4$. Now use the Hölder Inequality

$$
\int\left(\frac{f_{0}}{f_{n, j}}\right)^{1-\alpha} f_{n, j} \leq\left(\int\left(\frac{f_{0}}{f_{n, j}}\right)^{1 / 2} f_{n, j}\right)^{2(1-\alpha)}
$$

(since $2(1-\alpha)<1)$ to conclude that $h_{\alpha}\left(f_{0}, f_{n, j}\right) \leq\left[h_{1 / 2}\left(f_{0}, f_{n, j}\right)\right]^{2(1-\alpha)}$. Moreover, $f_{n, j} \in A_{j}$ implies that $h_{\alpha}\left(f_{0}, f_{n, j}\right)<\left(1-\epsilon^{2} / 4\right)^{2(1-\alpha)}<\mathrm{e}^{-\epsilon^{2}(1-\alpha) / 2}$. Now, similar to the proof of Theorem 1 , we obtain that

$$
\mathrm{P}\left(\sum_{j \geq 1} L_{n, j}^{\alpha}>\mathrm{e}^{-n d}\right)<\mathrm{e}^{n d} \mathrm{e}^{-n \epsilon^{2}(1-\alpha) / 2} \sum_{j \geq 1} \Pi\left(A_{j}\right)^{\alpha} .
$$

Thus condition (B.7) implies that $\sum_{j \geq 1} L_{n, j}^{\alpha}<\mathrm{e}^{-n d} F_{0}^{\infty}$-a.s. for all large $n$ and for any $d<\epsilon^{2}(1-\alpha) / 2$. On the oder hand, the Kullback-Leibler property ( $\mathbf{3 . 5}$ ) ensures that, for all large $n$ and for any $c>0, I_{n}^{\alpha} \geq \mathrm{e}^{-n \alpha c} F_{0}^{\infty}$-a.s.. Therefore

$$
\Pi_{n}\left(A_{1 / 2, \epsilon^{2} / 2}\right) \leq \sum_{j \geq 1} \frac{L_{n, j}^{\alpha}}{I_{n}^{\alpha}} \leq \mathrm{e}^{-n(d-\alpha c)} \rightarrow 0, \quad F_{0}^{\infty}-\text { a.s. }
$$

by taking $c$ sufficiently smaller than $\epsilon^{2}(1-\alpha) / 2 \alpha$.

\section{References}

Barron, A., Schervish, M. J. and Wasserman, L. (1999). The consistency of posterior distributions in nonparametric problems. Ann. Statist. 27, 536-561.

Berk, R. H. (1966). Limiting behaviour of posterior distributions when the model is incorrect. Ann. Math. Statist. 37, 51-58. [Corrigendum 37, 745-746]

Bingham, N. H., Goldie, C. M. and Teugels, J. L. (1987). Regular Variation, Cambridge University Press, Cambridge, UK.

Bunke, O. and Milhaud, X. (1998). Asymptotic behavior of Bayes estimates under possibly incorrect models. Ann. Statist. 26, 617-644. 
Ghosal, S., Ghosh, J. K. and Ramamoorthi, R. V. (1999). Posterior consistency of Dirichlet mixtures in density estimation. Ann. Statist. 27, 143-158.

Ghosal, S. and Roy, A. (2006). Posterior consistency of Gaussian process prior for nonparametric binary regression. Ann. Statist. 34, 2413-2429.

Kleijn, B. J. K. and van der Vaart, A. W. (2006). Misspecification in infinite-dimensional Bayesian statistics. Ann. Statist. 34, 837-887.

Lenk, P. J. (1998). The logistic normal distribution for Bayesian, nonparametric, predictive densities. J. Amer. Statist. Assoc. 83, 509-516.

Lenk, P. J. (1991). Towards a practicable Bayesian nonparametric density estimator. Biometrika 78, 531-543.

Leonard, T. (1978). Density estimation, stochastic processes and prior information (with discussion). J. Roy. Statist. Soc. Ser. B 40, 113-146.

Liese, F. and Vajda, I. (1987). Convex Statistical Distances. Teubner, Leipzig.

Liese, F. and Vajda, I. (2006). On divergence and informations in statistics and information theory. IEEE Trans. Inform. Theory 52, 4394-4412.

Lijoi, A., Prünster, I. and Walker, S. G. (2005). On consistency of nonparametric normal mixtures for Bayesian density estimation. J. Amer. Statist. Assoc. 100, 1292-1296.

Patilea, V. (2001). Convex models, MLE and misspecification. Ann. Statist. 29, 94-123.

Pfanzagl, J. (1990). Large deviation probabilities for certain nonparametric maximum likelihood estimators. Ann. Statist. 18, 1868-1877.

Petrone, S. and Wasserman, L. (2002). Consistency of Bernstein polynomial posteriors. J. $R$. Stat. Soc. Ser. B 64, 79-100.

Rockafellar, R. T. and Wets, R. J.-B. (2009). Variational Analysis. 3rd printing. Springer-Verlag, Berlin.

Shalizi, C. R. (2009). Dynamics of Bayesian updating with dependent data and misspecified models. Electron. J. Stat. 3, 1039-1074,

Tokdar, S. and Ghosh, J. (2007). Posterior consistency of Gaussian process priors in density estimation. J. Statist. Plann. Inference 137, 34-42.

van der Vaart, A. W. and Wellner, J. A. (1996). Weak Convergence and Empirical Processes. Springer, New York.

van der Vaart, A. W. and van Zanten, J. H. (2008). Rates of contraction of posterior distributions based on Gaussian process priors. Ann. Statist. 36, 1435-1463.

Walker, S. G. (2004). New approaches to Bayesian consistency. Ann. Statist. 32, 2028-2043.

Walker, S. G., Lijoi, A. and Prünster, I. (2005). Data tracking and the understanding of Bayesian consistency. Biometrika 92, 765-778.

Department of Economics and Statistics, University of Torino, Corso Unione Sovietica 218/bis, 10134 Torino, Italy.

E-mail: pierpaolo.deblasi@unito.it

Institute of Mathematics, Statistics and Actuarial Science, University of Kent, Kent CT2 7NZ, United Kingdom.

E-mail: S.G.Walker@kent.a..uk

(Received October 2010; accepted November 2011) 